\title{
Sports hernia: A pictorial review
}

\begin{abstract}
Author:
Peter Mercouris ${ }^{1}$

Affiliation:

${ }^{1}$ Lake, Smit and Partners,

Durban, South Africa

Correspondence to:

Peter Mercouris

Email:

pmercouris@iafrica.com

Postal address:

580 Currie Road, Durban

4001, South Africa

Dates:

Received: 11 June 2014

Accepted: 22 Sept. 2014

Published: 11 Dec. 2014

How to cite this article:

Mercouris, P. Sports hernia:

A pictorial review. S Afr J

Rad. 2014;18(2); Art. \#670,

4 pages. http://dx.doi.

org/10.4102/sajr.v18i2.670

\section{Copyright:}

C 2014. The Authors. Licensee: AOSIS

OpenJournals. This work is licensed under the Creative

Commons Attribution

License.
\end{abstract}

Read online:
Sports hernia represents a complex clinical and imaging entity. The purpose of the review is to provide a practical imaging approach to this condition. The review includes an overview of the anatomy and biomechanics of sports hernia by means of colour illustrations. The role of imaging, and particularly magnetic resonance imaging, is highlighted.

\section{Introduction}

Athletic pubalgia is a general term to describe exertional pubic or groin pain. ${ }^{1}$ Groin pain in elite athletes is common and accounts for approximately $5 \%-18 \%$ of all athletic injuries and is especially prevalent in kicking sports. ${ }^{2}$ The differential diagnosis is broad but the most common causes are considered to be adductor longus injury, common adductor-rectus abdominis dysfunction, osteitis pubis and sports hernia. ${ }^{1}$ This review is of the imaging of sports hernia.

\section{Definition}

Sports hernia represents an acquired deficiency of the inguinal wall and has been described by a myriad of names, such as Gilmore's groin, groin disruption, pubalgia, sportsman's hernia, prehernia complex, incipient hernia, symphysis syndrome and inguinal canal disruption. ${ }^{2}$ Practically, it is best appreciated as a deficiency that involves the anterior inguinal wall (external oblique muscle and aponeurosis), the posterior inguinal wall (transversus abdominis and internal oblique muscles), or both. Anterior inguinal wall deficiency represents an injury of the external oblique muscle aponeurosis, whereas posterior inguinal wall deficiency represents an injury at the level of the transversus abdominis and internal oblique muscles and aponeuroses. ${ }^{2}$

\section{Clinical presentation}

Athletes give a history of an insidious onset of groin pain that is initially experienced at the end of a game, progressing to pain during play and eventually pain at rest. Some may also experience pain at night. ${ }^{3}$ On physical examination, point tenderness may be elicited at or near the pubic symphysis, and there may be resistance or pain on ipsilateral hip adduction. Classically, there is no palpable hernia on physical examination. ${ }^{4}$

\section{Anatomy}

Anatomically, the anterior aspect of the pubis acts as a common origin for important musculotendinous, aponeurotic and ligamentous structures around the symphysis pubis (Figure 1). This prepubic soft-tissue complex is known as the prepubic aponeurotic complex (P-PAC) (Figure 2) and includes interconnections between the adductor tendons, rectus abdominis, inguinal musculoaponeurotic structures, articular disc and pubic ligaments of the symphysis pubis. ${ }^{1}$ Laterally, the caudal rectus abdominis lies just posteromedial to the superficial inguinal ring, and probably plays a role in reinforcing the wall of the posteromedial superficial inguinal canal. ${ }^{5}$

\section{Biomechanics}

The rectus abdominis and adductor longus are relative antagonists during core rotation and extension, with the result that the former elevates the anterior pelvis and the latter depresses it (Figure 3). ${ }^{3}$ There is therefore a dynamic imbalance between the strong adductors and weak abdominal musculature, resulting in shearing forces across the inguinal wall musculoaponeurotic and symphyseal structures (Figure 3).

\section{Imaging}

The fascial structures of the inguinal canal are not reliably imaged and sports hernia is therefore a clinical diagnosis and a radiological diagnosis of exclusion. ${ }^{2}$ Imaging in affected 


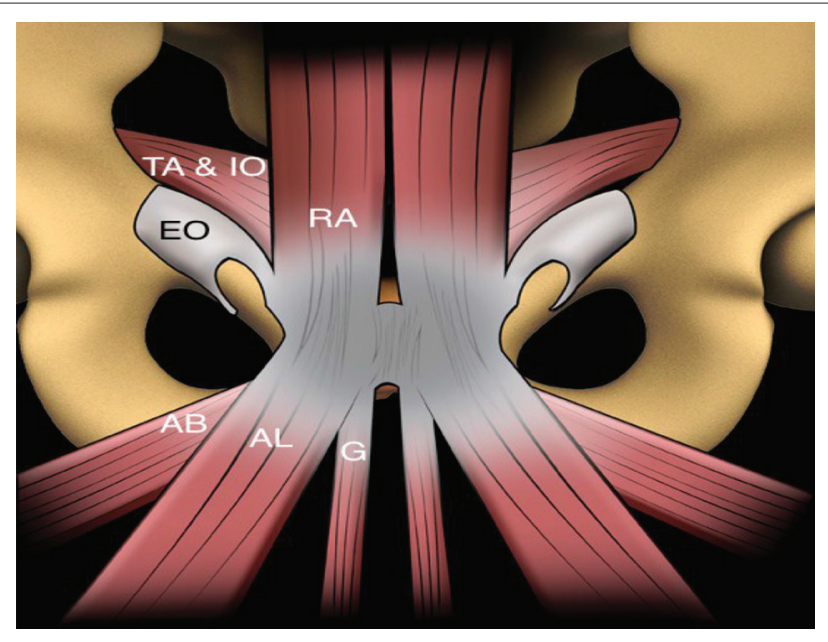

FIGURE 1: Musculotendinous and aponeurotic attachments of the anterior pubis $A B$, adductor brevis; $A L$, adductor longus; $E O$, external oblique aponeurosis; $G$, gracilis; IO, internal oblique; RA, rectus abdominis; TA, transversus abdominis.

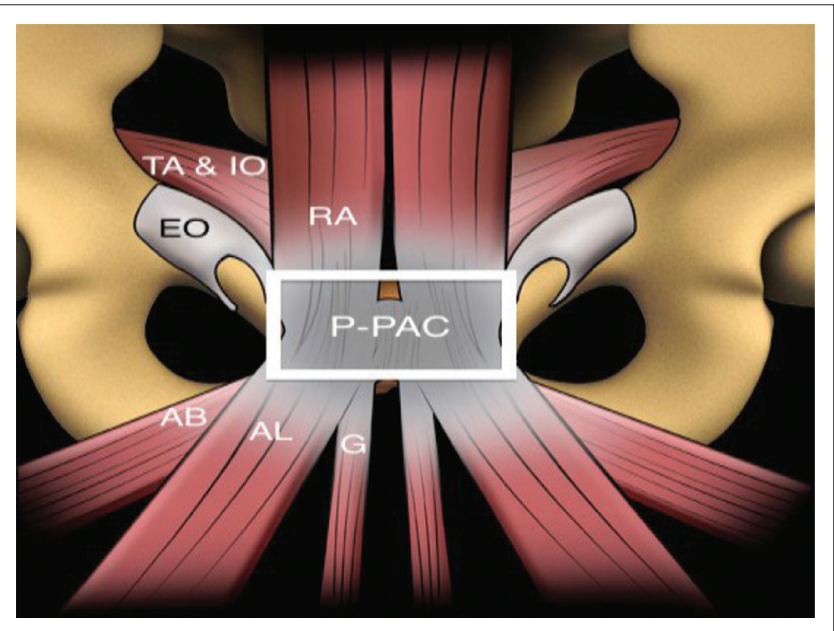

FIGURE 2: Prepubic aponeurotic complex (P-PAC).

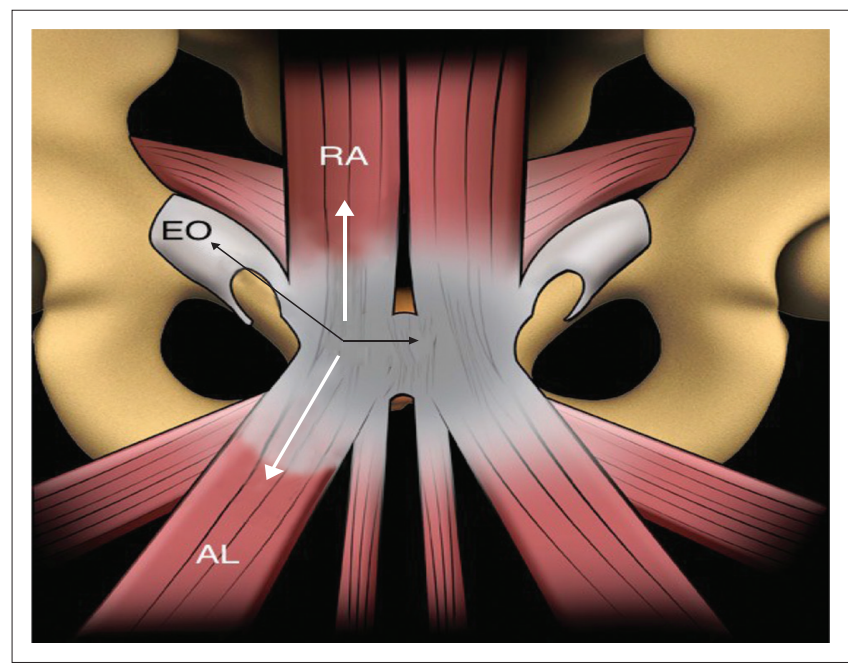

FIGURE 3: Vector and shearing forces across the prepubic aponeurotic complex (P-PAC). White arrows: vector forces; black arrows: shearing forces.

athletes is usually normal but occasionally injury to the inguinal aponeurotic structures can be identified. Imaging is, however, still important to exclude injury to the other

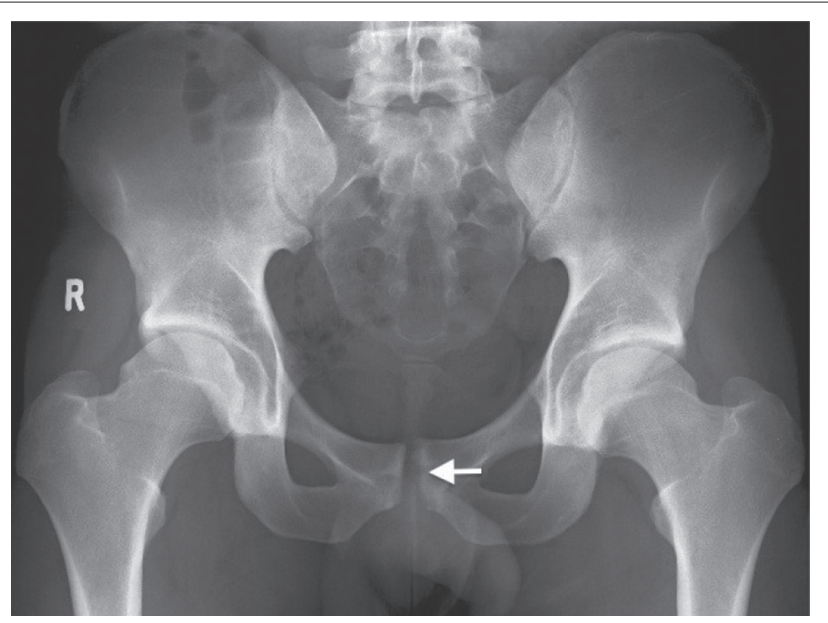

FIGURE 4: Osteitis pubis. Pelvic radiograph in a 21-year-old soccer player presenting with left groin pain demonstrating symphyseal cortical enthesiopathic changes on the left (white arrow). See Figure 6 for correlative MRI. There is also incidental cam-type femoro-acetabular impingement morphology.

musculoaponuerotic structures that constitute the prepubic aponeurotic complex.

The imaging approach of athletic pubalgia and sports hernia includes primarily the use of radiography, ultrasound and magnetic resonance imaging (MRI), with computed tomography (CT) and nuclear medicine imaging playing ancillary roles.

Obtaining a pelvic radiograph is still a good imaging principle to follow, although this is usually normal. A pelvic radiograph is useful in demonstrating symphyseal alignment and may indicate a potential osseous cause for the groin pain including hip, sacro-iliac, lower lumbar spine, and symphyseal (Figure 4) and apophyseal pathology.

Ultrasound, as with radiography, is also usually normal. Ultrasound is accurate in evaluating true inguinal and femoral hernias but these are rare in athletes. ${ }^{3}$ There is perhaps a role for dynamic ultrasound in evaluating potential posterior inguinal wall deficiency. This application arose from a study amongst Australian footballers in the late 1990s that documented the presence of abnormal ballooning of the posterior wall during straining. This sign was considered to be more specific if bilateral and in older athletes. ${ }^{6}$ Current consensus appears to indicate that this sign is too nonspecific and nonsensitive for widespread practical use. ${ }^{3}$

The goal of imaging is to accurately evaluate the musculoaponeurotic structures of the groin and is best achieved by MRI. MRI is both sensitive (98\%) and specific $(89 \%-100 \%)$ in patients with athletic pubalgia for injuries involving the rectus abdominis (Figure $5 \mathrm{~d}$ ), the adductor tendon origin (Figure 5), and the symphysis (Figure 6) itself.7 MRI is usually normal in sports hernia but an injury to the anterior (Figure 7 and Figure 8) or posterior wall (Figure 9) can sometimes be identified (Table 1). MRI is also useful in evaluating the regional osseous structures, as stress fractures (Figure 10) are common causes of groin pain beyond the inguinal and P-PAC regions (Table 1). 

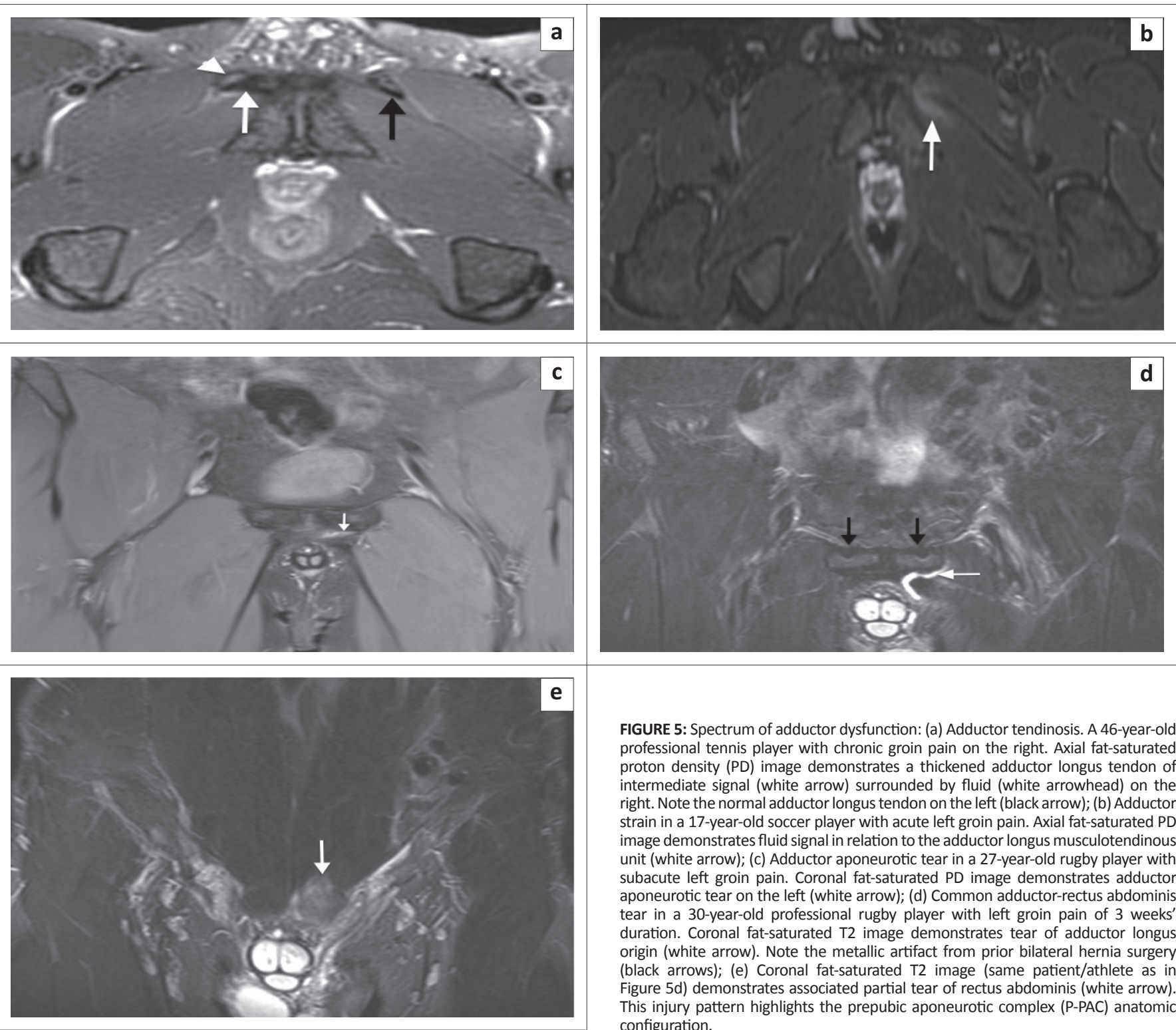

FIGURE 5: Spectrum of adductor dysfunction: (a) Adductor tendinosis. A 46-year-old professional tennis player with chronic groin pain on the right. Axial fat-saturated proton density (PD) image demonstrates a thickened adductor longus tendon of intermediate signal (white arrow) surrounded by fluid (white arrowhead) on the right. Note the normal adductor longus tendon on the left (black arrow); (b) Adductor strain in a 17-year-old soccer player with acute left groin pain. Axial fat-saturated PD image demonstrates fluid signal in relation to the adductor longus musculotendinous unit (white arrow); (c) Adductor aponeurotic tear in a 27-year-old rugby player with subacute left groin pain. Coronal fat-saturated PD image demonstrates adductor aponeurotic tear on the left (white arrow); (d) Common adductor-rectus abdominis tear in a 30-year-old professional rugby player with left groin pain of 3 weeks' duration. Coronal fat-saturated T2 image demonstrates tear of adductor longus origin (white arrow). Note the metallic artifact from prior bilateral hernia surgery (black arrows); (e) Coronal fat-saturated T2 image (same patient/athlete as in Figure $5 \mathrm{~d}$ ) demonstrates associated partial tear of rectus abdominis (white arrow). This injury pattern highlights the prepubic aponeurotic complex (P-PAC) anatomic configuration.
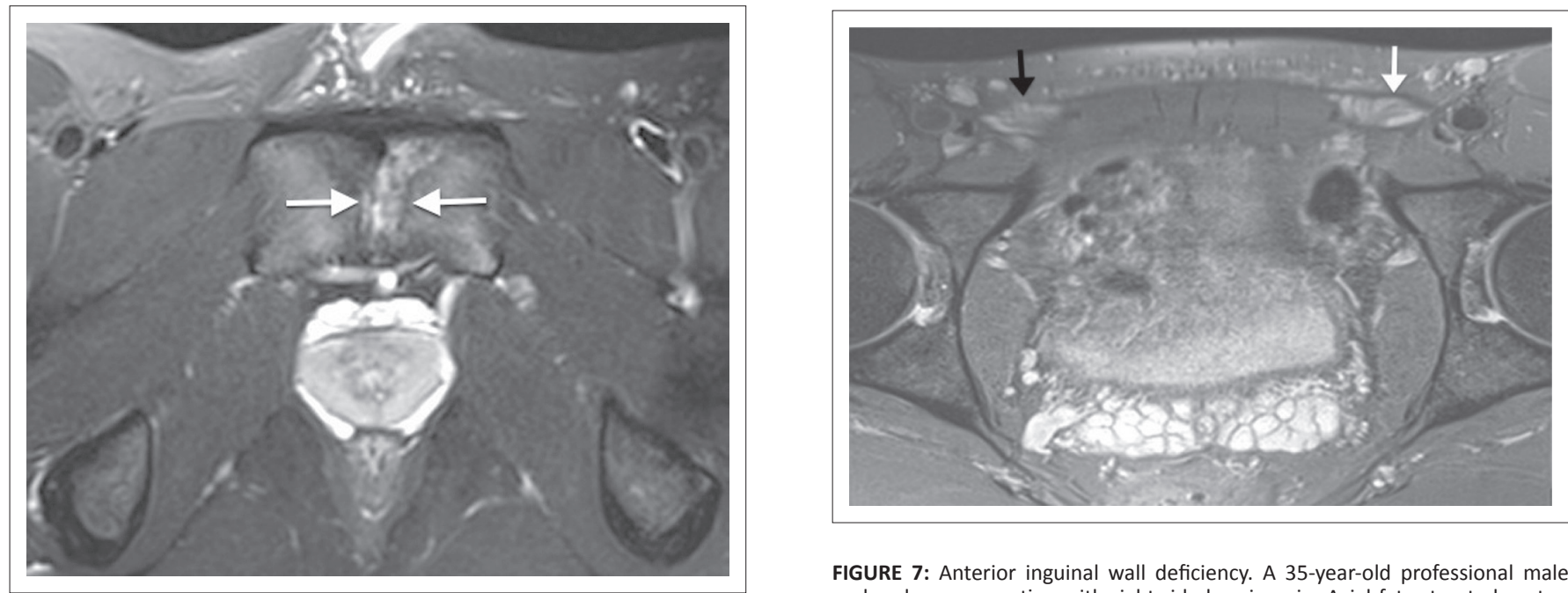

FIGURE 6: Osteitis pubis in a 21-year-old soccer player with left groin pain. Axia fat-saturated proton density (PD) image demonstrates bilateral and asymmetric bone marrow oedema in the symphysis pubis (white arrows). The hallmark of osteitis pubis is that the bone marrow oedema extends through the entire sagittal dimension of the bone as seen on the left in this athlete. See Figure 4 for correlative pelvic radiograph.

FIGURE 7: Anterior inguinal wall deficiency. A 35-year-old professional male rugby player presenting with right-sided groin pain. Axial fat-saturated proton density (PD) image demonstrates an external oblique aponeurosis tear on the right (black arrow) that was surgically confirmed. Note the intact aponeurosis on the left (white arrow). 


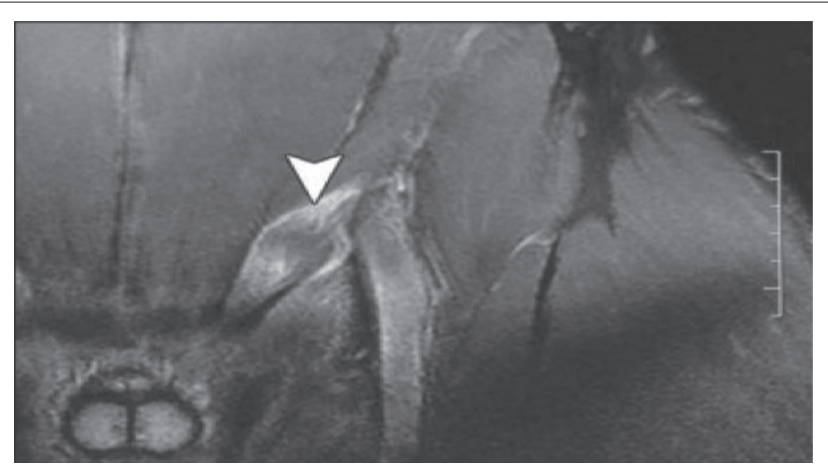

FIGURE 8: Anterior inguinal wall deficiency. Coronal T2-weighted fat-saturated image through the anterior abdominal wall of an elite 28-year-old male Australian-rules football player shows area of increased hyperintensity of left external inguinal ring, consistent with traumatic disruption of most medial fibre of the external oblique aponeurosis (arrowhead) and therefore in keeping with acute disruption of anterior inguinal wall. (Reprinted with permission from the American Journal of Roentgenology).

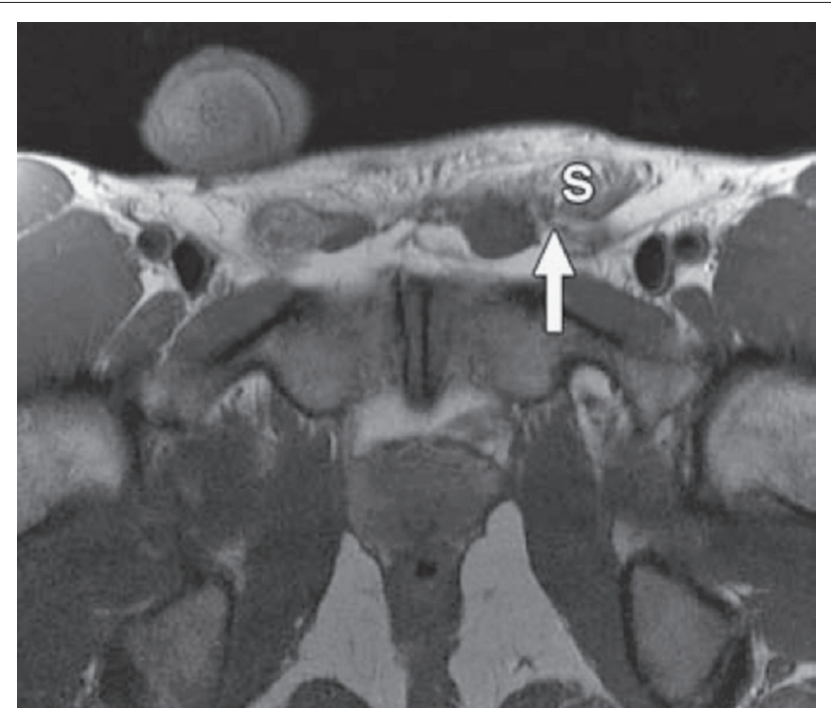

Source: Reprinted with permission from the American Journal of Roentgenology

FIGURE 9: Posterior inguinal wall deficiency. Axial proton density-weighted image in a 26-year-old male recreational soccer player with left-sided groin pain shows anterior bulging of posterior inguinal wall on left side (arrow), consistent with posterior inguinal wall deficiency. This results in a more anteriorly positioned spermatic cord (S) when compared with right side.

\section{TABLE 1: Sports hernia - key concepts.}

\section{Sports herni}

- Inguinal wall deficiency

- Clinical diagnosis

- Imaging is usually normal

- US to assess posterior inguinal wall integrity

- MRI to assess musculoaponeurotic structures of inguinal canal and P-PAC

MRI, magnetic resonance imaging; US, Ultrasound; P-PAC, prepubic aponeurotic complex.

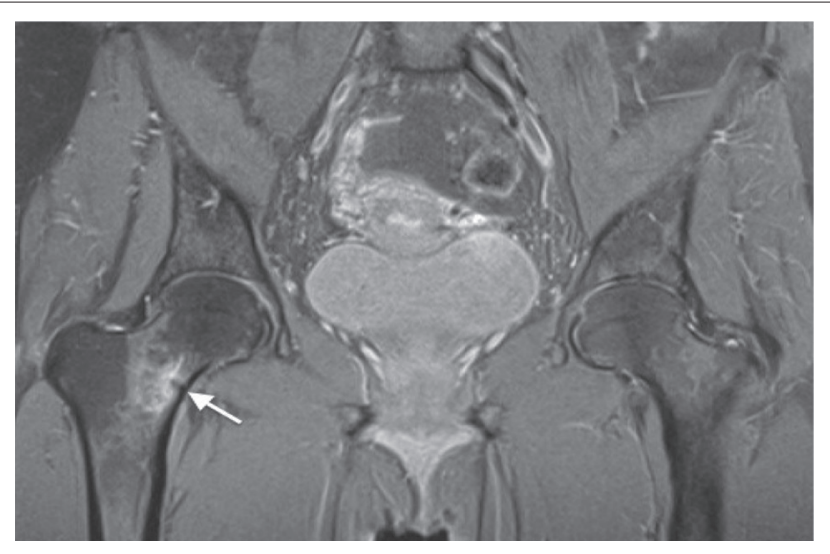

FIGURE 10: Stress fracture in a 38-year-old female runner with right groin pain. Coronal fat-saturated PD image demonstrates bone marrow oedema of the right femoral neck with an associated fracture (white arrow).

\section{Conclusion}

Sports hernia represents an acquired deficiency of the inguinal wall. Imaging is usually normal in affected athletes, and the entity remains a clinical diagnosis.

\section{Acknowledgements}

I thank Neil Northey for the medical illustrations.

\section{Competing interests}

The author declares that he has no financial or personal relationship(s) that may have inappropriately influenced him in writing this article.

\section{References}

1. MacMahon PJ, Hogan BA, Shelley MJ, Eustace SJ, Kavanagh EC. Imaging of groin pain. Magn Reson Imaging Clin N Am. 2009;17(4):655-666. http://dx.doi. org/10.1016/j.mric.2009.06.013

2. Koulouris G. Imaging review of groin pain in elite athletes: An anatomic approach to imaging findings. Am J Roentgenol. 2008;191(4):962-972. http://dx.doi. org/10.2214/AJR.07.3410

3. Robinson $P$, Bhat V, English B. Imaging in the assessment and management of athletic pubalgia. Semin Musculoskelet Radiol. 2001;15(1):14-26. http://dx.doi. org/10.1055/s-0031-1271956

4. Zajick DC, Zoga AC, Omar IM, Meyers WC. Spectrum of MRI findings in clinical athletic pubalgia. Semin Musculoskelet Radiol. 2008:12(1):3-12. http://dx.dol. org/10.1055/s-2008-1067933

5. Khan W, Zoga AC, Meyers WC. Magnetic resonance imaging of athletic pubalgia and sports hernia. Current understanding and practice. Magn Reson Imaging Clin N Am. 2013;21(1):97-110. http://dx.doi.org/10.1016/j. mric.2012.09.008

6. Orchard JW, Read JW, Neophyton J, Garlick D. Groin pain associated with ultrasound finding of inguinal canal posterior wall deficiency in Australian Rules footballers. Br J Sports Med. 1998;32(2):134-139.

7. Zoga AC, Kavanagh EC, Omar IM, et al. Athletic pubalgia and the 'sports hernia': MR imaging findings. Radiology. 2008;247(3):797-807. http://dx.doi.org/10.1148/ radiol. 2473070049 